\title{
Front Matter: Volume 9575
}

, "Front Matter: Volume 9575," Proc. SPIE 9575, Optical Manufacturing and Testing XI, 957501 (15 September 2015); doi: 10.1117/12.2208522

EDent: SPIE Optical Engineering + Applications, 2015, San Diego, California, SPIE. United States 


\title{
PROCEEDINGS OF SPIE
}

\section{Optical Manufacturing and Testing XI}

\author{
Oliver W. Fähnle \\ Ray Williamson \\ Dae Wook Kim \\ Editors
}

9-11 August 2015

San Diego, California, United States

Sponsored and Published by

SPIE 
The papers in this volume were part of the technical conference cited on the cover and title page. Papers were selected and subject to review by the editors and conference program committee. Some conference presentations may not be available for publication. Additional papers and presentation recordings may be available online in the SPIE Digital Library at SPIEDigitallibrary.org.

The papers reflect the work and thoughts of the authors and are published herein as submitted. The publisher is not responsible for the validity of the information or for any outcomes resulting from reliance thereon.

Please use the following format to cite material from this proceedings:

Author(s), "Title of Paper," in Optical Manufacturing and Testing XI, edited by Oliver W. Fähnle, Ray Williamson, Dae Wook Kim, Proceedings of SPIE Vol. 9575 (SPIE, Bellingham, WA, 2015) Six-digit Article CID Number.

ISSN: 0277-786X

ISSN: 996-756X (electronic)

ISBN: 9781628417418

Published by

SPIE

P.O. Box 10, Bellingham, Washington 98227-0010 USA

Telephone +1 3606763290 (Pacific Time) · Fax +1 3606471445

SPIE.org

Copyright (C) 2015, Society of Photo-Optical Instrumentation Engineers.

Copying of material in this book for internal or personal use, or for the internal or personal use of specific clients, beyond the fair use provisions granted by the U.S. Copyright Law is authorized by SPIE subject to payment of copying fees. The Transactional Reporting Service base fee for this volume is $\$ 18.00$ per article (or portion thereof), which should be paid directly to the Copyright Clearance Center (CCC), 222 Rosewood Drive, Danvers, MA 01923. Payment may also be made electronically through CCC Online at copyright.com. Other copying for republication, resale, advertising or promotion, or any form of systematic or multiple reproduction of any material in this book is prohibited except with permission in writing from the publisher. The CCC fee code is 0277-786X/15/\$18.00.

Printed in the United States of America.

Publication of record for individual papers is online in the SPIE Digital Library.

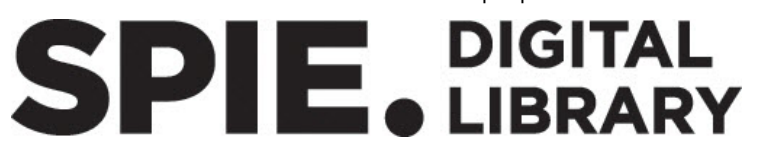

SPIEDigitalLibrary.org

Paper Numbering: Proceedings of SPIE follow an e-First publication model, with papers published first online and then in print. Papers are published as they are submitted and meet publication criteria. A unique citation identifier (CID) number is assigned to each article at the time of the first publication. Utilization of CIDs allows articles to be fully citable as soon as they are published online, and connects the same identifier to all online, print, and electronic versions of the publication. SPIE uses a six-digit CID article numbering system in which:

- The first four digits correspond to the SPIE volume number.

- The last two digits indicate publication order within the volume using a Base 36 numbering

system employing both numerals and letters. These two-number sets start with 00, 01, 02, 03, 04, $05,06,07,08,09,0 A, 0 B \ldots$. OZ, followed by 10-1Z, 20-2Z, etc.

The CID Number appears on each page of the manuscript. The complete citation is used on the first page, and an abbreviated version on subsequent pages. 


\title{
Contents
}

\author{
vii Authors \\ ix Conference Committee
}

\section{SESSION 1 OPTICAL SYSTEMS I}

957502 Examination of the quality of $\mathbf{1 2 0}$ degree silicon double mirror for a micro-optical laser gyroscope [9575-1]

957504 JWST pathfinder telescope integration [9575-3]

957505 JWST pathfinder telescope risk reduction cryo test program [9575-4]

\section{SESSION 2 OPTICAL SYSTEMS II}

957506 Optomechanical design and tolerance of a microscope objective at 121.6 nm [9575-5]

957508 Metrology requirements for the serial production of ELT primary mirror segments [9575-7]

\section{SESSION 3 OPTICAL MANUFACTURING I}

957509 Surface roughness when diamond turning RSA 905 optical aluminium [9575-10]

9575 OA Aspects of ultra-high-precision diamond machining of RSA443 optical aluminium [9575-11]

$9575 \mathrm{OB}$ The role of robotics in computer controlled polishing of large and small optics [9575-12]

9575 OC Evaluation and control of spatial frequency errors in reflective telescopes [9575-13]

9575 OD Correction of mid-spatial-frequency errors by smoothing in spin motion for CCOS [9575-57]

\section{SESSION 4 OPTICAL MANUFACTURING II}

$9575 \mathrm{OH} \quad$ Fabrication of freeform optics [9575-17]

9575 0J Measuring skew in average surface roughness as a function of surface preparation [9575-49] 
9575 OL Status of the Advanced Mirror Technology Development (AMTD) phase 2, 1.5m ULE ${ }^{\circledR}$ mirror [9575-21]

$95750 \mathrm{M} \quad$ Improving profitability through slurry management: a look at the impact of slurry $\mathrm{pH}$ on various glass types [9575-22]

9575 ON Material removal characteristics of orthogonal velocity polishing tool for efficient fabrication of CVD SiC mirror surfaces [9575-23]

957500 Surface errors in the course of machining precision optics [9575-24]

9575 OP The effect of deep HF etching on the surface quality and figure of fused silica optics [9575-59]

\section{SESSION 6 OPTICAL MANUFACTURING IV}

$95750 Q \quad$ Using frictional power to model LSST removal with conventional abrasives [9575-26]

9575 OR Influence of coolant on ductile mode processing of binderless nanocrystalline tungsten carbide through ultraprecision diamond turning [9575-27]

9575 OS Influence of temperature difference on surface figure controlling during continuous polishing [9575-60]

\section{SESSION 7 OPTICAL TESTING I}

9575 OW Subaperture stitching surface errors due to noise [9575-32]

9575 0X An iterative subaperture position correction algorithm [9575-33]

\section{SESSION $8 \quad$ OPTICAL TESTING II}

957511 Measuring and quantifying scatter from non-isotropic sources [9575-36]

957512 Fabrication and qualification of roughness reference samples for industrial testing of surface roughness levels below $0.5 \mathrm{~nm}$ Sq [9575-37]

\section{SESSION 9 OPTICAL TESTING III}

957514 Oil defect detection of electrowetting display [9575-40]

957515 Deflectometry measurement of Daniel K. Inouye Solar Telescope primary mirror [9575-41]

957516 The use of diffractive imitator optics as calibration artefacts [9575-42] 
957519 Investigation of rapidly solidified aluminum by using diamond turning and a magnetorheological finishing process [9575-45]

95751 A Optical diffraction interpretation: an alternative to interferometers [9575-46]

9575 1C Noninvasive method for determination of parameters of cemented doublet [9575-48]

9575 ID Detection of subsurface defects and measurement of thickness of screen layers made of graphene and carbon nanotubes with application of full-field optical coherence tomography in Linnik configuration [9575-50]

$95751 G$ Designing null phase screens to test a fast plano-convex aspheric lens [9575-53]

$9575 \mathrm{1H} \quad$ Null screens type Hartmann to test simple lenses [9575-54] 
Proc. of SPIE Vol. $9575957501-6$

Downloaded From: https://www.spiedigitallibrary.org/conference-proceedings-of-spie on 25 Apr 2023 Terms of Use: https://www.spiedigitallibrary.org/terms-of-use 


\section{Authors}

Numbers in the index correspond to the last two digits of the six-digit citation identifier (CID) article numbering system used in Proceedings of SPIE. The first four digits reflect the volume number. Base 36 numbering is employed for the last two digits and indicates the order of articles within the volume. Numbers start with 00, 01, 02, 03, 04, 05, 06, 07, 08, 09, 0A, 0B...0Z, followed by 10-1Z, 20-2Z, etc.

Abou-El-Hossein, Khaled, 09, 0A, 19

Allen, Richard G., $0 Q$

Amon, John N., 04

Asfour, Jean-Michel, 16

Atkinson, Charles B., 04

Audo, F., lA

Avendaño-Alejo, Maximino, 1G, 1H

Bibby, Matt, OB

Biskup, H., 0O, 12

Blalock, Todd, $\mathrm{OH}$

Boffa, Christopher C., OM

Bouillet, S., 1A

Broccolo, Ronald T., 04

Burge, James H., OW, 15

Castán-Ricaño, Diana, 1G, 1H

Castillo-Santiago, Gabriel, $1 \mathrm{H}$

Chen, Yi-Chun, OX

Cheng, Yuan-Chieh, 09, 19

Chiang, Hou-Chi, 14

Cumme, M., 12

Cureton, Kevin, OM

Daly, Elizabeth A., 04

Dambon, Olaf, OR

Daurios, J., $1 \mathrm{~A}$

DeGroote Nelson, Jessica, $\mathrm{OH}$

DelOlmo-Márquez, Jesús, $1 G$

Densmore, Victor, 06

Díaz-Uribe, Rufino, 1G, 1H

Dietzel, Andreas, 02

Doetz, Marius, OR

Dun, Aihuan, OS

Dunn, Christina, OB

Effinger, Michael R., OL

Egerman, Robert, OL

Ellis, James M., 04

Eng, Ron, OL

Eupherte, L., $1 \mathrm{~A}$

Fähnle, Oliver, OR, 12

Fechner, R., 12

Feinberg, Lee D., 04, 05

Ferland, Albert, OL

Fréville, S., 1A

Frost, F., 12

Gao, Weichuan, 06

Gao, Wenlan, OP, OS

Gozález-Galindo, Alfredo, 1H

Gray, Caroline, 08

Gu, Haojin, OD

Haberl, A., 00
Hadaway, James B., 05

Hahn, Walter G., 04

Han, Jeong-Yeol, ON

Havey, Keith A., Jr., 05

Hong, Meijuan, OS

Hooper, Abigail R., OM

Hsu, Wei-Yao, 09, 19

$\mathrm{Hu}$, Chen, OD

$\mathrm{Hu}$, Haixiang, $\mathrm{OC}$

Huang, Run, 15

Huang, Ting-Wei, 14

Hubler, William H., $\mathrm{OQ}$

Huguet, Jesse A., 05

Johnson, Matthew, OL

Jota, Thiago S., 06

Kennard, Scott H., 04

Keski-Kuha, Ritva, 04, 05

Keyes, Derek S., 06

Kim, Gun-Hee, 06

Kim, Sug-Whan, ON

Kim, Young-Sik, 06

Klocke, Fritz, OR

Kuo, Ching-Hsiang, 19

Lander, Juli A., 05

Langenbach, E., 12

Leber, Ingmar, 02

Lee, Haengbok, ON

Lee, Kyungmook, ON

Levi, Joshua, 04

Li, Hongyu, OB

Lin, Po-Chih, OX

Liu, Shijie, OD, OP, OS

Lo, Weng-Hou, OX

Lu, Chunlian, OB

Luepke, Dakota, 06

Lunt, Sharon, 05

Mang, Ou-Yang, 14

Marsh, James M., 05

Matthews, Gary W., 04, 05, 0L

McKay, Andrew, 04

Medicus, Kate, $\mathrm{OH}$

Mikš, A., $1 \mathrm{C}$

Milster, Thomas D., 06

Mitchell, John B., 16

Mkoko, Z., 09, 0A

Mlynarska, Paulina, 1D

Mt. Pleasant, Stephen M., 04

Nasyrov, Ruslan K., 16

Niesel, Thalke, 02 
Noël, Darin M., 05

Novák, J., $1 \mathrm{C}$

Novák, P., 1C

Otieno, Timothy, 09, 19

Pakuła, Anna, 1D

Pokorný, P., 1C

Poleshchuk, Alexander G., 16

Rascher, R., 00, 12

Rees, Paul C. T., 08, 16

Rouyer, C., IA

Salbut, Leszek, 1D

Sarkas, Harry W., OM

Schindler, A., 12

Scorse, Thomas R., 05

Seo, Hyunju, ON

Seong, Sehyun, ON

Shao, Jianda, OD, OP, OS

Sloma, Marcin, 1D

Smith, Greg A., OW

Spina, John A., 05

Stahl, H. Philip, OL

Stahl, Mark T., OJ

Stover, John C., 11

Su, Peng, 15

Texter, Scott, 04

Tomczewski, Slawomir, 1D

Tsai, Yu-Hsiang, 14

Volkov, Andy, 16

Voyton, Mark F., 05

Walker, Chanda B., 05

Walker, David, OB

Wei, Chaoyang, OD, OP, OS

Weidner, Frank, 16

Wells, Conrad, 05

Whitman, Tony L., 05

Wróblewski, Grzegorz, 1D

Wu, Hsing Yu, OB

Wünsche, C., 12

$X U$, Jiafeng, $O P$

Xu, Xueke, OD, OP, OS

Yan, Yung-Jhe, 14

Yang, Minghong, OP, OS

Yoon, Siyoung, ON

Yu, Guoyu, OB

Zeng, Xuefeng, OC

Zhang, Haichao, OD

Zhang, Xuejun, OC

Zhang, Yang, OS

Zhang, Yizhong, OD

Zheng, Ligong, OC

Zheng, Xiao, OB

Zygalsky, F., 12 


\section{Conference Committee}

Program Track Chair

H. Philip Stahl, NASA Marshall Space Flight Center (United States)

Conference Chairs

Oliver W. Fähnle, FISBA OPTIK AG (Switzerland)

Ray Williamson, Ray Williamson Consulting (United States)

Dae Wook Kim, College of Optical Sciences, The University of Arizona

(United States)

Conference Program Committee

Haobo Cheng, Tsinghua University (China)

Olaf Dambon, Fraunhofer-Institut für Produktionstechnologie

(Germany)

Peter J. de Groot, Zygo Corporation (United States)

Jessica E. DeGroote Nelson, Optimax Systems, Inc. (United States)

Richard R. Freeman, Zeeko Ltd. (United Kingdom)

Roland Geyl, Sagem Défense Sécurité (France)

John E. Greivenkamp, College of Optical Sciences, The University of Arizona (United States)

Steve E. Kendrick, Ball Aerospace \& Technologies Corporation

(United States)

Sugwhan Kim, Yonsei University (Korea, Republic of)

Sven R. Kiontke, asphericon GmbH (Germany)

Cody B. Kreischer, Kreischer Optics, Ltd. (United States)

Gary W. Matthews, Exelis Visual Information Solutions (United States)

Robert E. Parks, Optical Perspectives Group, LLC (United States)

Rolf Rascher, Hochschule Deggendorf (Germany)

Joseph L. Robichaud, L-3 Communications SSG-Tinsley (United States)

Joanna Schmit, Bruker Corporation (United States)

Sven Schröder, Fraunhofer-Institut für Angewandte Optik und

Feinmechanik (Germany)

Shai N. Shafrir, Corning Incorporated (United States)

Tayyab I. Suratwala, Lawrence Livermore National Laboratory (United States)

Flemming Tinker, Aperture Optical Sciences Inc. (United States)

Martin J. Valente, College of Optical Sciences, The University of Arizona (United States)

David D. Walker, Zeeko Research Ltd. (United Kingdom)

Konrad Wegener, ETH Zürich (Switzerland)

Christine Wünsche, Hochschule Deggendorf (Germany) 
Takashi Yatsui, The University of Tokyo (Japan)

Xue-jun Zhang, Changchun Institute of Optics, Fine Mechanics and Physics (China)

\section{Session Chairs}

1 Optical Systems I

Dae Wook Kim, College of Optical Sciences, The University of Arizona (United States)

2 Optical Systems II

Ray Williamson, Ray Williamson Consulting (United States)

3 Optical Manufacturing I

Oliver W. Fähnle, FISBA OPTIK AG (Switzerland)

4 Optical Manufacturing II

Jessica DeGroote Nelson, Optimax Systems, Inc. (United States)

5 Optical Manufacturing III

Martin J. Valente, Arizona Optical Systems, LLC (United States)

6 Optical Manufacturing IV

David D. Walker, Zeeko Research Ltd. (United Kingdom)

7 Optical Testing I

Ray Williamson, Ray Williamson Consulting (United States)

8 Optical Testing II

Xue-jun Zhang, Changchun Institute of Optics, Fine Mechanics and Physics (China)

9 Optical Testing III

Ray Williamson, Ray Williamson Consulting (United States) 